\title{
DVCS and Vector Meson Production with H1
}

\author{
Pierre Marage \\ Faculté des Sciences - Université Libre de Bruxelles \\ ULB-CP 230 - Boulevard du Triomphe - B-1050 Bruxelles - Belgium
}

\begin{abstract}
Recent results on Deeply Virtual Compton Scattering (DVCS) and exclusive vector meson (VM) production obtained with the H1 detector at HERA are reviewed. Emphasis is put on the transition from soft to hard diffraction and on spin dynamics [1].
\end{abstract}

\section{Introduction}

Studies of the DVCS process and of exclusive VM production at HERA provide, in the presence of a hard scale, unique information on the mechanism of diffraction, in particular on the transition from soft to hard diffraction and spin dynamics.

Exclusive final states studied by the H1 collaboration include real photons $[2,3]$ and light $(\rho$ and $\phi[4,5,6])$ and heavy $\operatorname{VMs}(J / \psi[7,8], \psi(2 s)[9]$ and $\Upsilon[10])$, in the elastic and proton dissociation channels. A hard scale is provided by the VM mass $M_{V}$, by the negative square of the photon four-momentum, $Q^{2}$ (with $Q^{2} \simeq 0$ for photoproduction and $1.5 \leq Q^{2} \leq 90 \mathrm{GeV}^{2}$ for electroproduction), or by the square of the four-momentum transfer at the proton vertex, $t$. Cross sections are expressed in terms of $\gamma^{*} p$ scattering.

The data are interpreted in terms of two complementary QCD approaches. Following a collinear factorisation theorem, the electroproduction of light VMs by longitudinally polarised photons and the production of heavy VMs can be described by the convolution of the hard process with generalised parton distributions in the proton (GPDs). High energy DVCS and VM production can also be described as the factorisation of virtual photon fluctuation into a $q \bar{q}$ colour dipole, diffractive dipole-proton scattering, and $q \bar{q}$ recombination into the final state photon or VM. The interaction scale $\mu$ is given by the characteristic transverse size of the dipole, with $\mu^{2} \simeq\left(Q^{2}+M_{V}^{2}\right) / 4$ for $J / \psi$ production and light VM production by longitudinal photons, whereas this value may be significantly reduced for light VM production by transversely polarised photons, because of end-point contributions in the photon wave function. For DVCS, the role of LO contributions support a scale of the order of $Q^{2}$. Several models based on either approach are compared to the H1 data; references and brief descriptions can be found in $[2,5]$.

High $|t|$ photoproduction data with $2 \leq|t| \leq 30 \mathrm{GeV}^{2}[3,6,8]$, which are not further presented here, tend to support BFKL evolution.

\section{Kinematic dependences}

DVCS The $Q^{2}$ evolution of DVCS production [2], presented in Fig. 1, is well described by models both using GPDs or a dipole approach. The interference of the DVCS and Bethe-Heitler processes gives access, through the measurement of beam charge asymmetry, to the ratio $\rho$ of the real to imaginary parts of the DVCS amplitude. The measurement is $\rho=0.20 \pm 0.05 \pm 0.08$, in agreement with the value $\rho=0.25 \pm 0.03 \pm 0.05$ obtained from a dispersion relation using the $W$ dependence of the cross section.

DIS 2009 

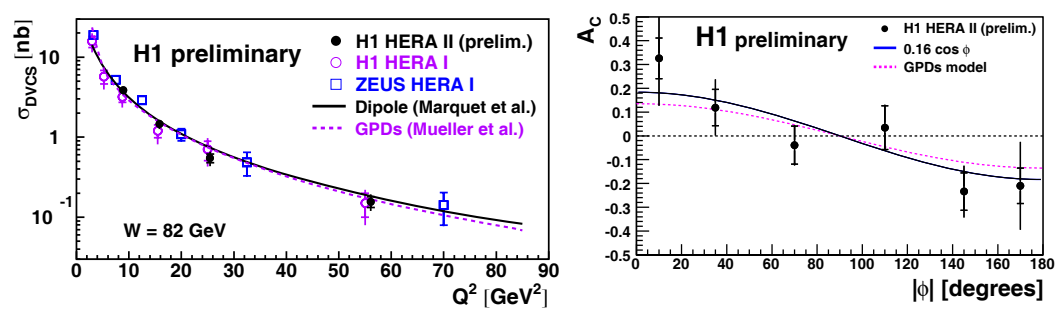

Figure 1: (left) $Q^{2}$ dependence of DVCS production and model predictions; (right) beam charge asymmetry, $\cos \phi$ fit and predictions of a GPD model [2].

Light VM electroproduction The cross sections for elastic and proton dissociative $\rho$ and $\phi$ electroproduction have been measured with high precision [5]. The $Q^{2}$ dependence, shown for $\rho$ mesons in Fig. 2, is reasonably described by several models, which predict separately the longitudinal and transverse cross sections (for polarised cross sections, see [5]).

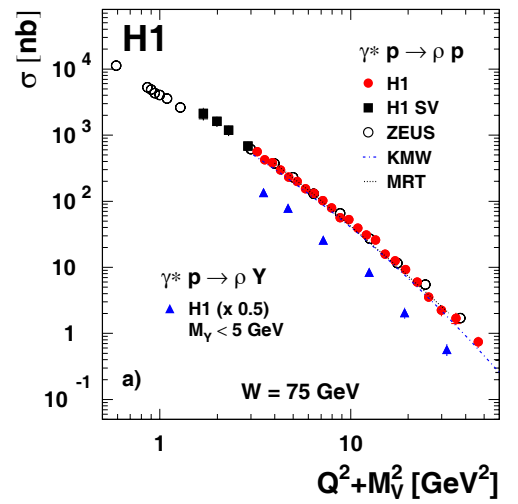

Figure 2: $Q^{2}$ dependence of elastic and proton dissociative electroproduction cross sections of $\rho$ mesons, with model predictions [5].

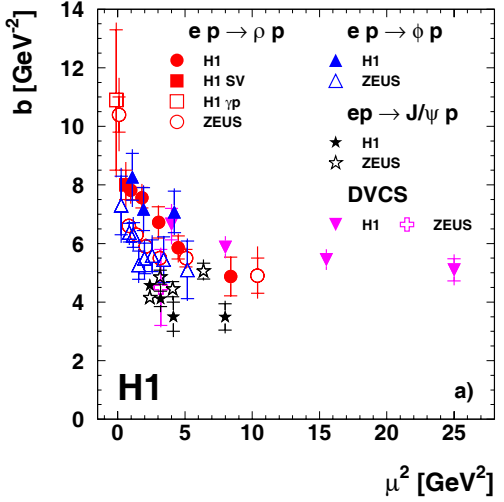

Figure 3: Elastic $b$ slopes, as a function of $\mu^{2}=\left(Q^{2}+M_{V}^{2}\right) / 4$ for $\mathrm{VM}$ production and $\mu^{2}=Q^{2}$ for DVCS.

$t$ slopes The $|t|$ distributions for DVCS, light and heavy VM production, both in the elastic channel with $|t| \leq 0.5 \mathrm{GeV}^{2}$ and in the proton dissociative channel with $|t| \lesssim 2.5 \mathrm{GeV}^{2}$, are exponentially falling, with $\mathrm{d} \sigma / \mathrm{dt} \propto \mathrm{e}^{-\mathrm{b}|\mathrm{t}|}$. For each process, the slope $b$ is given by the convolution of the transverse sizes of the $q \bar{q}$ dipole, of the diffractively scattered system (which vanishes for proton dissociation) and of the exchange (expected to be small). As observed in Fig. 3, the elastic slopes for light VMs strongly decrease with increasing $Q^{2}$, reflecting dipole shrinkage. Values similar to those for $J / \psi$ production are reached for $\left(Q^{2}+M_{V}^{2}\right) / 4 \gtrsim 5 \mathrm{GeV}^{2}$. A similar evolution is observed for DVCS as a function of $Q^{2}$.

The difference between elastic and proton dissociative slopes, $b_{e l}-b_{p . d i s s .}$, provides a test of proton vertex or "Regge" factorisation. It is of $3.5 \pm 0.1 \mathrm{GeV}^{-2}$ for $J / \psi$, with a similar value for DVCS. The difference is higher, around 5.5, for $\rho$ and $\phi$ mesons, with however an indication of a decrease toward the $J / \psi$ value with increasing $\left(Q^{2}+M_{V}^{2}\right) / 4$. 
Energy dependence and effective Regge trajectory The energy dependence of DVCS and VM production is well described by a power law, $\mathrm{d} \sigma / \mathrm{dW} \propto \mathrm{W}^{\delta}$. For $J / \psi$ photoproduction, the value of $\delta$ is significantly larger than for (soft) hadron-hadron interactions, with $\delta \sim 1.2$. This confirms that $J / \psi$ photoproduction is a hard process, characterised by small transverse dipoles, which probes the low- $x$ gluon density in the proton at a scale where it is quickly increasing. For light VMs, the $W$ dependence is hardening with $Q^{2}$, and $\delta$ reaches $J / \psi$ values for $\left(Q^{2}+M_{V}^{2}\right) / 4 \gtrsim 5 \mathrm{GeV}^{2}$.
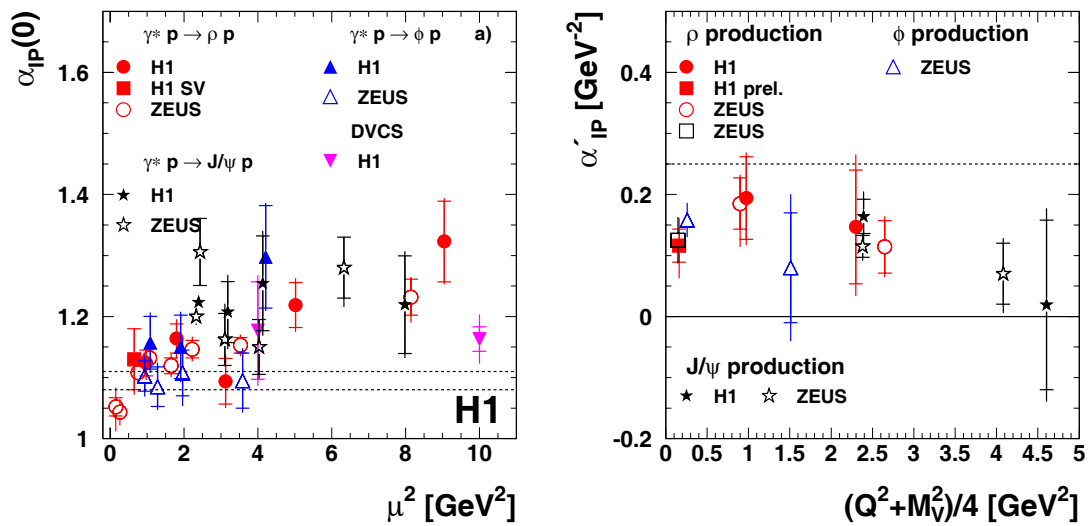

Figure 4: Measurement of (left) the intercept $\alpha_{\mathbb{P}}(0)$ and (right) the slope $\alpha^{\prime}$ of the effective Regge trajectory, as a function of the scale $\mu^{2}=\left(Q^{2}+M_{V}^{2}\right) / 4$ for VM production and $\mu^{2}=Q^{2}$ for DVCS.

In a Regge inspired parameterisation, the energy dependence of the cross section and its correlation with $t$ are given by $\delta(t)=4\left(\alpha_{\mathbb{P}}(t)-1\right)$, with $\alpha_{\mathbb{P}}(t)=\alpha_{\mathbb{P}}(0)+\alpha^{\prime} \cdot t$, where $\alpha^{\prime}$ describes the shrinking of the diffractive peak with energy. The hard behaviour of $J / \psi$ production and the hardening with $\left(Q^{2}+M_{V}^{2}\right) / 4$ of the energy dependence of light VM production for $t=0$ is demonstrated in Fig. 4, where values of 1.08 or 1.11 for $\alpha_{\mathbb{P}}(0)$ are typical of soft hadron-hadron interactions. The slope of the effective trajectory for VM production, including $\rho$ photoproduction [4], is smaller than the typical value $0.25 \mathrm{GeV}^{-2}$ in hadronic interactions. For DVCS $\alpha^{\prime}=0.03 \pm 0.09 \pm 0.11 \mathrm{GeV}^{-2}[2]$; for $J / \psi$ photoproduction at high $|t|$, combining $\mathrm{H} 1[8]$ and ZEUS measurements, $\alpha^{\prime}=-0.02 \pm 0.01 \pm 0.01 \mathrm{GeV}^{-2}$.

\section{$3 \quad$ Helicity amplitudes}

The VM production and decay angular distributions allow the measurement of fifteen spin density matrix elements, which are bilinear combinations of helicity amplitudes. Under natural parity exchange, five $T_{\lambda_{V} \lambda_{\gamma}}$ amplitudes are independent: two $s$-channel helicity conserving (SCHC) amplitudes $\left(T_{00}\right.$ and $\left.T_{11}\right)$, two single helicity flip amplitudes ( $T_{01}$ and $\left.T_{10}\right)$ and one double flip amplitude $\left(T_{-11}\right)$.

The $Q^{2}$ dependence of the matrix elements for $\rho$ production is presented in Fig. 5. The five elements which contain products of the SCHC amplitudes are non-zero, whereas those formed with the helicity violating amplitudes are generally consistent with 0 . A notable exception is the element $r_{00}^{5}$, which involves the product of the dominant $T_{00} \mathrm{SCHC}$

DIS 2009 

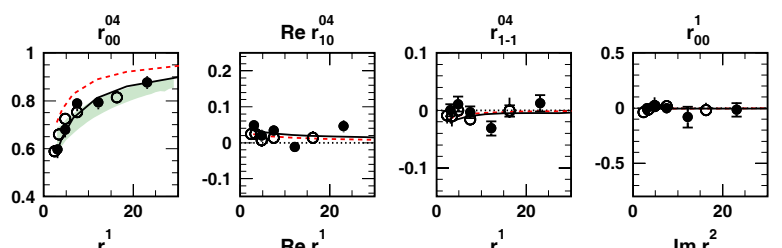

$\operatorname{Re} \mathbf{r}_{10}^{1}$
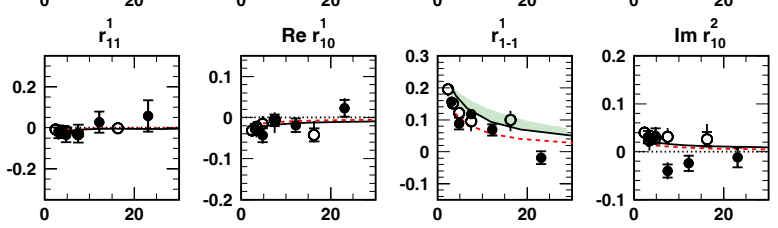

$\operatorname{Im} \mathrm{r}_{1-1}^{2}$

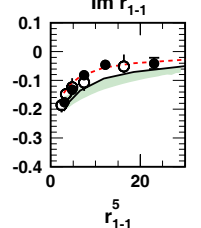

$\mathbf{r}_{00}^{5}$
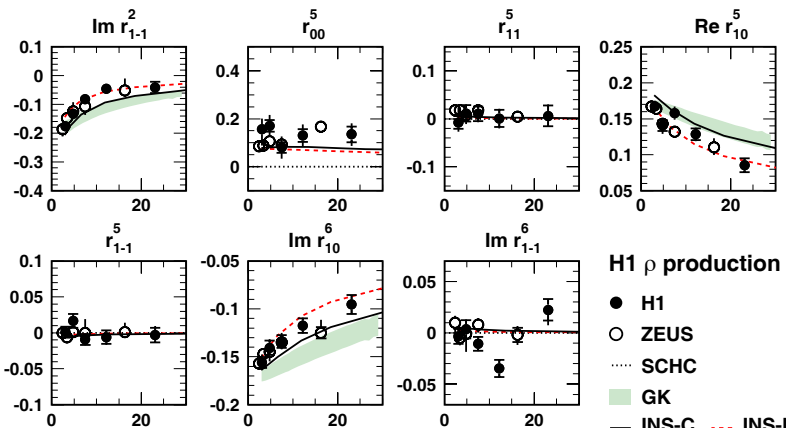

H1 $\rho$ production

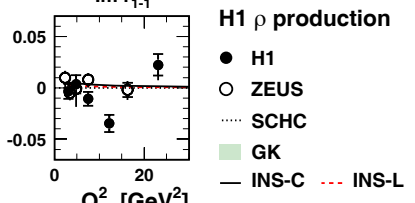

Figure 5: $Q^{2}$ dependence of the spin density matrix elements for $\rho$ electroproduction; model predictions are superimposed. The dotted lines show the expected vanishing values if only the SCHC amplitudes are non-zero [5].

amplitude with $T_{01}$, which describes the transition from a transversely polarised photon to a longitudinal $\rho$ meson.

The ratio $R=\sigma_{L} / \sigma_{T}$ of the longitudinal to transverse cross sections for $\rho$ production is shown is Fig. 6 as a function of $Q^{2}$ and, for two domains in $Q^{2}$, as a function of $t$ and the invariant mass of the two decay pions.

A strong increase of $R$ with $Q^{2}$ is observed, which is tamed at large $Q^{2}$. These features are relatively well described by models. The $Q^{2}$ dependence of $R$ for $\rho, \phi$ and $J / \psi$ VMs follows a universal trend when plotted as a function of $Q^{2} / M_{V}^{2}$ [5].

An increase of $R$ with $t$ is observed for $Q^{2}>5 \mathrm{GeV}^{2}$. This can be translated into a measurement of the difference between the longitudinal and transverse $t$ slopes, through the relation $R(t)=\sigma_{L}(t) / \sigma_{T}(t) \propto$ $e^{-\left(b_{L}-b_{T}\right)|t|}$. A slight indication $(1.5 \sigma)$ is thus found for a negative value of $b_{L}-b_{T}\left(-0.65 \pm 0.14_{-0.51}^{+0.41}\right)$ suggesting that, as expected, the average transverse size of dipoles for transverse amplitudes is larger than for longitudinal amplitudes
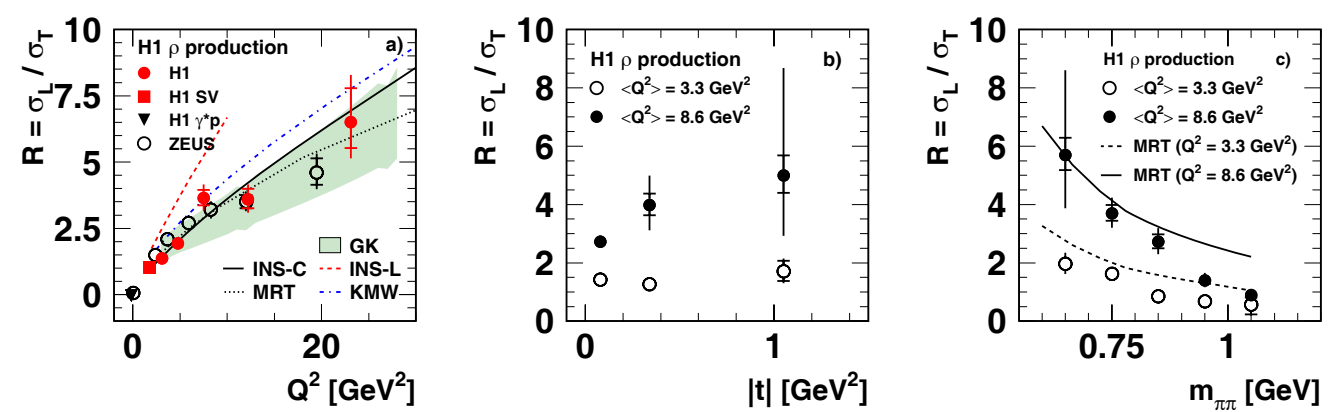

Figure 6: Measurement of $R=\sigma_{L} / \sigma_{T}$, as a function of $Q^{2}, t$ and the invariant mass of the two decay pions, for $\rho$ electroproduction [5]; model predictions are superimposed.

The dependence of $R$ with the dipion mass, which can not be attributed solely to the interference of resonant $\rho$ and non-resonant $\pi \pi$ production, indicates that the spin dynamics

DIS 2009 
of $\rho$ production depends of the effective $q \bar{q}$ mass. No dependence of $R$ in $W$ is observed with the present data.
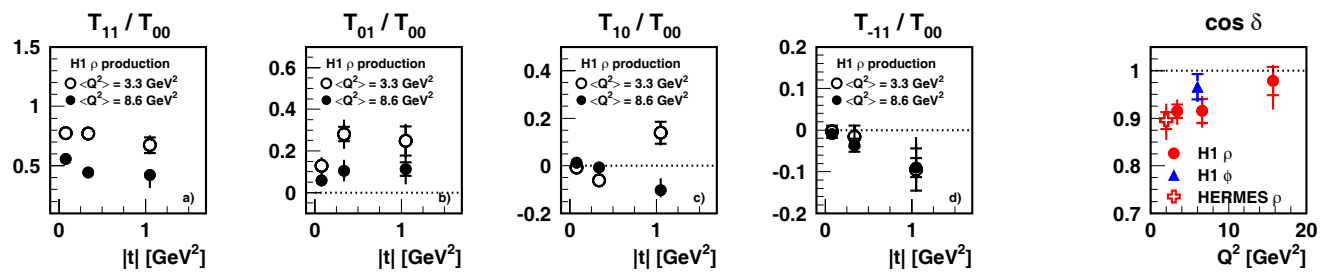

Figure 7: (a-d) Helicity amplitude ratios, as a function of $t$; (right plot) phase difference between the two SCHC amplitudes, $T_{00}$ and $T_{11}$ [5].

Helicity amplitude ratios are measured, under the approximation that they are in phase, through fits to the 15 matrix elements. The four ratios to the dominant $T_{00}$ amplitude are presented in Fig. 7 as a function of $t$, for two domains in $Q^{2}$. At large $Q^{2}$, a $t$ dependence compatible with the expected $\sqrt{|t|}$ law is observed for both single helicity flip amplitudes. A significant double-flip amplitude $T_{-11}$ is observed, which may be related to gluon polarisation in the proton. The $t$ dependence of $T_{11} / T_{00}$ at large $Q^{2}$, a $3 \sigma$ effect, is related to the $t$ dependence of $R$ and supports the indication of a difference between the transverse size of dipoles in transversely and longitudinally polarised photons.

A small non-zero phase difference between the two SCHC amplitudes, which decreases with increasing $Q^{2}$, is visible in Fig. 7. Through dispersion relations, this non-zero value is suggestive of different $W$ dependences of the longitudinal and transverse amplitudes.

\section{Acknowledgments}

It is a pleasure to thank numerous colleagues from the ZEUS and H1 collaborations as well as theorists for enlightening discussions, and the workshop organisers and session conveners for the lively discussions and the pleasant atmosphere of the meeting.

\section{References}

[1] Slides of the talk: http://indico. cern. ch/contributionDisplay py? contribId=184\&sessionId=25\&conf Id=53294

[2] H1 Collab., DVCS and its Beam Charge Asymmetry in ep collisions at HERA, H1-prelim-09-014.

[3] F.D. Aaron et al. [H1 Collab.], Phys. Lett. B672 219 (2009); T. Hreus, these proc., arXiv:0907.2317 [hep-ex].

[4] H1 Collab., A Measurement of the Pomeron Trajectory based on Elastic $\rho$ Photoproduction, H1-prelim09-016 (2009); B. List, these proc., arXiv:0906.4945 [hep-ex].

[5] F.D. Aaron et al. [H1 Collab.], Diffractive electroproduction of $\rho$ and $\phi$ mesons, DESY-09-093 (2009).

[6] A. Aktas et al. [H1 Collab.], Phys. Lett. B638 422 (2006).

[7] A. Aktas et al. [H1 Collab.], Eur. Phys. J. C46 585 (2006).

[8] A. Aktas et al. [H1 Collab.], Phys. Lett. B568 205 (2003).

[9] C. Adloff et al. [H1 Collab.], Phys. Lett. B541 251 (2002).

[10] C. Adloff et al. [H1 Collab.], Phys. Lett. B483 23 (2000).

DIS 2009 